\title{
Comparisons of VLP-Based ELISA, Neutralization Assays with Native HPV, and Neutralization Assays with PsV in Detecting HPV Antibody Responses in HIV-Infected Women
}

\author{
Ping Du ${ }^{1,2 *}$, Sarah Brendle ${ }^{3}$, Janice Milici ${ }^{4}$, Fabian Camacho ${ }^{2}$, John Zurlo ${ }^{1}$, Neil Christensen ${ }^{3}$ and Craig Meyers $^{4}$ \\ ${ }^{1}$ Department of Medicine, The Pennsylvania State University College of Medicine, USA \\ ${ }^{2}$ Department of Public Health Sciences, The Pennsylvania State University College of Medicine, USA \\ ${ }^{3}$ Department of Pathology, The Pennsylvania State University College of Medicine, USA \\ ${ }^{4}$ Department of Microbiology and Immunology, The Pennsylvania State University College of Medicine, USA
}

\begin{abstract}
Objective: Human papillomavirus (HPV)-associated cancers are important public health problems in HIV-infected people. Assays based on HPV virus-like particles (VLP) and pseudoviruses (PsV) are commonly used to examine HPV antibody responses in HIV-infected people, but neutralization assays with native HPV have not been utilized and a comparison of these three assays is lacking. We evaluated the agreement of assays using VLP, native HPV and PsV in detecting HPV16 and 18 antibodies in HIV-infected women.
\end{abstract}

Methods: The VLP-based ELISA (VLP-ELISA) was used to detect antibody responses to HPV16 and 18 and cottontail rabbit papillomavirus (CRPV) VLP antigens. Neutralization assays with native HPV (NA-HPV) and with PsV (NA-PsV) were conducted to examine HPV16 or 18 neutralizing antibodies. Intra class correlation coefficients (ICC) and kappa coefficients were used to assess the agreements of seropositivity between the assays.

Results: The seroprevalence detected by the VLP-ELISA, NA-HPV and NA-PsV in 94 HIV-infected women was $35 \%, 51 \%$ and $27 \%$ for HPV 16 and $14 \%, 44 \%$ and $21 \%$ for HPV 18 . Cross-reactivity between HPV 16 and HPV 18 was $0.35,0.04$ and 0.33 (kappa coefficients) for the VLP-ELISA, NA-HPV and NA-PsV. The agreements of seropositivity between the three assays were low. Six women who were HPV16 DNA positive were seropositive by the NA-HPV but only two were HPV16 seropositive by the VLP-ELISA or NA-PsV. One HPV18 DNA positive woman was seropositive by all three assays. Repeated tests indicated excellent reproducibility of the NA-HPV.

Conclusion: HPV serology results vary across different assays. The NA-HPV appears to be a sensitive and reliable approach in detecting natural HPV antibodies in HIV-infected women. The NA-HPV can be applied in both HPV natural history studies and vaccine studies in HIV-infected people.

Keywords: HIV-infected women; Human papillomavirus; Virus-like particles; ELISA; Pseudoviruses; Neutralization assay

\section{Introduction}

Human papillomavirus (HPV) infection is highly prevalent in people with human immunodeficiency virus (HIV) infection, and HPV-associated cancers are important public health problems in HIVinfected people $[1,2]$. Seroepidemiology is an essential method to assess host immunity following HPV infection [3]. Although native HPV can be cultivated in differentiated epithelial tissues using the organotypic (raft) culture system [4], there is a lack of utilization of native HPV as the antigens in population health research to assess antibody responses against HPV infection. Recombinant particles have been utilized as surrogates of native HPV; the commonly used recombinant particles include the virus-like particles (VLP) and pseudoviruses (PsV), which are produced by over-expression and self-assembly of HPV capsid proteins ( $\mathrm{L} 1$ or $\mathrm{L} 1+\mathrm{L} 2)[5,6]$. While the World Health Organization has established the reference standards of negative and positive reagents for antibodies to HPV16 $[7,8]$, there is no standard assay to detect HPV antibodies.

Different approaches have been utilized in previous research to examine antibody responses to HPV capsid proteins (L1 or L1+L2). Briefly, the HPV VLP-based assays, including the direct enzymelinked immunosorbent assay (ELISA) and the multiplex immunoassay systems, are widely used to measure antibodies against HPV typespecific L1 major capsid protein or the neutralizing epitopes, and certain cut-off values are established to determine the seropositivity of a serum sample [9-11]. The secreted alkaline phosphatase neutralization assay (SEAP-NA) with HPV PsV carrying a reporter gene has also been developed to measure the neutralization potential of HPV antibodies by blocking PsV from infecting the cells [12]. However, some studies have found that different assays yield different serology results, limiting the comparison of the results across studies and the choice of an assay for monitoring HPV antibody responses [13-20].

A high seroprevalence of HPV antibodies in HIV-infected women has been detected using the VLP-based assays [21-23], but neutralization assays have not been well conducted to examine the presence of HPV neutralizing antibodies in HIV-infected women. Advances in HPV immunology could have great implications for HPV-related cancer prevention in HIV populations [24]. In this exploratory study, we used

*Corresponding author: Ping Du, Department of Medicine, The Pennsylvania State University College of Medicine, Tel: (717) 531-4194; Fax: (717) 531-5779; E-mail: ping.du@psu.edu

Received January 14, 2015; Accepted February 20, 2015; Published February 27, 2015

Citation: Du P, Brendle S, Milici J, Camacho F, Zurlo J, et al. (2015) Comparisons of VLP-Based ELISA, Neutralization Assays with Native HPV, and Neutralization Assays with PsV in Detecting HPV Antibody Responses in HIV-Infected Women. J AIDS Clin Res 6: 433. doi:10.4172/2155-6113.1000433

Copyright: (c) $2015 \mathrm{Du} \mathrm{P}$, et al. This is an open-access article distributed under the terms of the Creative Commons Attribution License, which permits unrestricted use, distribution, and reproduction in any medium, provided the original author and source are credited. 
Citation: Du P, Brendle S, Milici J, Camacho F, Zurlo J, et al. (2015) Comparisons of VLP-Based ELISA, Neutralization Assays with Native HPV, and Neutralization Assays with PsV in Detecting HPV Antibody Responses in HIV-Infected Women. J AIDS Clin Res 6: 433. doi:10.4172/21556113.1000433

the VLP-based ELISA (VLP-ELISA), the neutralization assay with native HPV (NA-HPV), and the neutralization assay with PsV (NA$\mathrm{PsV}$ ) to evaluate HPV antibody responses in HIV-infected women and to assess the agreement of seropositivity between these assays.

\section{Materials and Methods}

HIV-infected women were recruited from 5 HIV/AIDS outpatient clinics in south central Pennsylvania during 2010 to 2012. Study participants provided oral, vaginal, and anal swabs and $10 \mathrm{ml}$ of whole blood. HIV/AIDS-related medical information was obtained from the electronic medical records. The swab specimens were tested for the presence of 37 types of HPV DNA (Roche Diagnostics, Indianapolis, IN), and the serum samples were used for HPV serology tests. The serum samples were heat-inactivated at $56^{\circ} \mathrm{C}$ for 30 minutes and stored at $-20^{\circ} \mathrm{C}$ prior to the tests. All the serum samples were tested using the VLP-ELISA, the NA-HPV, and the NA-PsV. To assess the reproducibility of the three assays, 9 serum samples were randomly chosen to repeat each assay. This study was approved by the Pennsylvania State University College of Medicine Institutional Review Board and written informed consent was obtained from each study participant. All work was performed in accordance with the ethical standards that guide biomedical research involving human subjects.

\section{HPV VLP-based ELISA (VLP-ELISA)}

Direct ELISAs were performed to assess serum type-specific antibodies (IgG+IgA+IgM) to HPV VLP antigens as previously described [10]. Because we found high background signals from all serum samples that would cause false positive results, we first coated the ELISA plate wells with $200 \mathrm{ng} /$ well of heparin conjugated to BSA, which was shown to reduce background in serum ELISAs [25]. Second we used the cottontail rabbit papillomavirus (CRPV) VLP antigens as a negative control to adjust for background reactivity in the serum samples [10]. Sera from previously reported virgins or sera from children is not a preferable negative control for these studies because our samples are from older, HIV-infected women and the possibility of finding HIV-infected women without HPV exposure is nearly impossible. The normal population will be negative against CRPV antigens as this virus grows on rabbits in the western United States and most people are not in contact with these animals. HPV16, 18, and CRPV L1 VLPs were produced in 293 TT cells and were used as the antigens $[5,6,9,26]$. CRPV VLP PBS-incubated wells were used as negative control wells. Each patient's serum and negative controls were tested at a final dilution of 1:100 in duplicate wells. The paired t-test was used (Sigmaplot 11, Systat Software Inc.) to compare the mean optical density (OD) A405/A490 values of the ELISA VLPs with the mean OD value of the serological reaction to the CRPV to determine HPV VLP antibody seropositivity [10]. If the mean VLP OD value is significantly higher than the mean CRPV OD value (at $\mathrm{p}$-value $<0.05$ ), the serum is defined as seropositive.

\section{Neutralization assays with native HPV (NA-HPV)}

The NA-HPV were conducted in HaCaT cells using the method that we developed before [27]. Briefly, native HPV16 and HPV18 were cultured using HPV16- or HPV18-persistently infected human foreskin keratinocyte (HFK) cell lines in the organotypic (raft) culture system $[4,27,28]$. Native HPV16 and HPV18 were then purified from the 20-day raft culture tissues [27]. Based on our previous experience, we chose a multiplicity of infection of 25 (MOI25) for the assay. The native HPV MOI25, patient serum (1:100 dilution) and media were combined and incubated at $37^{\circ} \mathrm{C}$ for one hour. A mixture of native virus and media without patient serum was used as the negative control. As a positive control for neutralization, we combined native HPV with previously characterized conformation dependent type-specific mouse monoclonal antibodies (mAbs) (HPV16.V5 and HPV18.J4) [10,29]. The mixtures were then placed on 175,000 adherent HaCaT cells in 24well plates and incubated at $37^{\circ} \mathrm{C}$ for 48 hours. The mRNAs of HPVinfected cells were harvested with the RNeasy Mini Kit Kit (Qiagen Inc., Valencia, CA). Real-time quantitative PCR (RT-qPCR) was performed using the QuantiTech Probe RT-PCR Kit (Qiagen Inc., Valencia, CA) to assess relative expression levels of $\mathrm{HPV}$ E1^${ }^{\wedge} \mathrm{E} 4$ transcripts based on the levels of the endogenous reference transcript (TBP). HPV16 and HPV18 primers and probes were developed using Gene Link Software (OligoAnalyzer 1.2 and OligoExplorer 1.2) [27]. The CT values of the mixtures were obtained and then transformed as the relative infectivity values using REST $\odot$ software to determine neutralization potential $[27,30]$. The relative infectivity value was set to 1.0 for the negative control. Based on the reduction in infectivity compared with the negative control, the seropositivity was defined as having a $\leq 0.5$ relative infectivity value (representing a $50 \%$ or more reduction in infectivity).

\section{Neutralization assays with PsV (NA-PsV)}

PsV16 and 18 encapsidating a secreting alkaline phosphatase plasmid were produced in 293TT cells and neutralization assays with PsV were performed in 293TT cells as previously described [6,12,31,32]. Briefly, 293TT cells were seeded in 96 well plates in DMEM without phenol red. The next day, approximately $1.6 \times 10^{5} \mathrm{PsV}$ particles were incubated with an indicated dilution of patient sera or monoclonal antibody $(\mathrm{mAb})$ for 1 hour at $37^{\circ} \mathrm{C}$ before adding to duplicate wells [31] Three days post-seeding, $30 \mu \mathrm{l}$ cell-culture supernatant was assayed with $p$-nitrophenylphosphate tablets (Sigma) dissolved in Tris buffer and secreted alkaline phosphatase signal was read at OD A405/A490. $\mathrm{A}$ mixture of PsV and media without patient serum was used as the negative control, and a mixture of PsV and mouse mAbs (H16.V5 and H18.J4, respectively) was used as the positive control for neutralization $[10,29]$. The seropositivity was defined using the same criterion for the NA-HPV (a $50 \%$ or more reduction in infectivity compared with the negative control).

\section{Assessment of non-specific neutralization}

To rule out possible non-specific neutralization, we also conducted the neutralization assays with native CRPV (NA-CRPV) in RK-13 cells MOI25 to examine non-specific neutralization, as antibodies to HPV should not neutralize CRPV. CRPVs were obtained from infected rabbit xenografts grown in athymic mice. RK-13 cells (rabbit keratinocytes) were grown in DMEM (10\% FBS) and $2.5 \times 10^{5}$ cells were seeded in 12 well plates. After 24 hours, CRPV and a 1:50 dilution of sera were mixed in $100 \mathrm{ul}$ culture media and incubated for 1 hour at $37^{\circ} \mathrm{C}$. Neutralizing monoclonal antibodies (CRPV.2C and CRPV.4B) were included as positive controls [33]. The following day $500 \mathrm{ul}$ culture media was added to wells and 24 hours later the media was removed and fresh media was added. After a total incubation time of 120 hours the cells were lysed and harvested with TRIzol (Invitrogen). Total RNA was isolated from TRIzol and RT-qPCR was performed to assess relative expression levels of $\mathrm{HPV} \mathrm{E} 1 \wedge \mathrm{E} 4$ transcripts [34,35]. A total of $400 \mathrm{ng}$ of RNA was assayed in duplicate multiplex reactions with TATA-binding protein (TBP) as the internal reference control using the Brilliant II QRT-PCR kit (Agilent Technologies) on the Mx-3005 (Agilent Technologies). All RT-qPCR data were analyzed using REST software [30]. The seropositivity was defined using the same criterion for the NA-HPV. All CRPV-seropositive samples were further diluted (1:100 and 1:200) to assess neutralization potential. 
Citation: Du P, Brendle S, Milici J, Camacho F, Zurlo J, et al. (2015) Comparisons of VLP-Based ELISA, Neutralization Assays with Native HPV, and Neutralization Assays with PsV in Detecting HPV Antibody Responses in HIV-Infected Women. J AIDS Clin Res 6: 433. doi:10.4172/21556113.1000433

\section{Statistical analysis}

The seroprevalence, the cross-reactivity between HPV16 and HPV18, and the reproducibility were examined for each assay. Because this study focused on the seropositivity as a dichotomous variable (Yes or No), Cohen's kappa coefficients were calculated to evaluate the cross-reactivity and the reproducibility. To assess the agreement and the consistency between the three assays, intraclass correlation coefficients (ICC) were calculated to distinguish the results that could be highly correlated but with little agreement, while kappa coefficients were used to rule out a random agreement due to chance.

\section{Results}

A total of $94 \mathrm{HIV}$-infected women were included in this study and none of them ever received HPV vaccines. Most of HIV-infected women in this study were older than 40 years (median age $=46$ years, age range: 20-66 years), non-Hispanic whites (52\%), and had more than 5 lifetime sexual partners $(68 \%)$. The majority of these women acquired HIV via heterosexual contact (74\%) and had been infected with HIV for more than 10 years $(67 \%)$. At the time of recruitment, $82 \%$ of women were taking combined antiretroviral therapy for HIV infection. About 55\% of women had an average CD4 counts $>500$ cells $/ \mathrm{mm}^{3}$ and $61 \%$ had low HIV RNA viral loads (undetectable at $\leq 75$ copies $/ \mathrm{mm}^{3}$ ). More than half of these women (54\%) had at least one abnormal cervical cytology (atypical squamous cells of undetermined significance or worse) in previous years, and two women were diagnosed with invasive cervical cancer in 2001 and 2003.

Overall, the seroprevalence detected by the VLP-ELISA, the NA$\mathrm{HPV}$, and the NA-PsV in $94 \mathrm{HIV}$-infected women was 35\%, 51\%, and
$27 \%$ for HPV16 antibody, and 14\%, 44\% and 21\% for HPV 18 antibody, respectively (Table 1). Kappa coefficients indicated a low cross-reactivity between HPV16 and HPV18 for the NA-HPV (kappa $=0.04,95 \%$ confidence interval: $-0.15,0.24)$, but a moderate cross-reactivity for the VLP-ELISA (kappa $=0.35$ ) and the NA-PsV (kappa $=0.33)$. HPV16 DNA was detected from oral, vaginal, or anal swabs in six women (6\%); all of these women were seropositive by the NA-HPV but only two were seropositive by the VLP-ELISA or the NA-PsV. One woman who was HPV18 DNA positive was seropositive by all three assays.

The serology results varied across the three assays (Table 2). The concordant seropositive or seronegative results were observed only among $34 \%$ of the samples for HPV16 and 53\% for HPV18. None of the assays could fully capture the seropositivity as some samples were only seropositive in one assay but not in other two assays. Both the agreement and the consistency of the results (measured by the kappa coefficients and the ICCs) were very low between these three assays (Table 3). There was a slight agreement between the neutralization assays with native HPV18 and PsV18 (kappa=0.29).

Four samples (4\%) showed seropositivity against CRPV in the NA-CRPV and were determined to have non-specific neutralization effects (at a 1:50 dilution). Of these four samples, one was seropositive with native HPV18, one was seropositive with native HPV16 and 18, one was seropositive with VLP16 and 18 and native HPV18, and one was seropositive with VLP16 and 18, native HPV18, and PsV16 and 18. The samples were then diluted to $1: 100$ and three samples still neutralized CRPV. When the samples were further diluted to 1:200, no neutralization effect was observed.

Nine random samples were repeated for each assay for HPV16

\begin{tabular}{|c|c|c|c|c|c|c|c|}
\hline & & \multicolumn{2}{|c|}{ VLP-ELISA* } & \multicolumn{2}{|c|}{ NA-HPV** } & \multicolumn{2}{|c|}{ NA-PsV** } \\
\hline & & \multicolumn{2}{|c|}{ HPV16 } & \multicolumn{2}{|c|}{ HPV16 } & \multicolumn{2}{|c|}{ HPV16 } \\
\hline & & Positive & Negative & Positive & Negative & Positive & Negative \\
\hline \multirow{2}{*}{ HPV18 } & Negative & 11 & 2 & 22 & 19 & 11 & 9 \\
\hline & Positive & 22 & 59 & 26 & 27 & 14 & 60 \\
\hline \multicolumn{8}{|c|}{ Seroprevalence } \\
\hline \multicolumn{2}{|c|}{ HPV16 } & \multicolumn{2}{|c|}{$35 \%$} & \multicolumn{2}{|c|}{$51 \%$} & \multicolumn{2}{|c|}{$27 \%$} \\
\hline \multicolumn{2}{|c|}{ HPV18 } & \multicolumn{2}{|c|}{$14 \%$} & \multicolumn{2}{|c|}{$44 \%$} & \multicolumn{2}{|c|}{$21 \%$} \\
\hline \multicolumn{8}{|c|}{ Cross-reactivity } \\
\hline \multicolumn{2}{|c|}{ Kappa coefficients $†$} & \multicolumn{2}{|c|}{$0.35(0.16,0.53)$} & \multicolumn{2}{|c|}{$0.04(-0.15,0.24)$} & \multicolumn{2}{|c|}{$0.33(0.11,0.54)$} \\
\hline
\end{tabular}

*Definition of seropositive for VLP-ELISA: If the mean VLP OD value is significantly higher than the mean CRPV OD value (at $p$-value<0.05), the serum is defined as seropositive.

**Definition of seropositive for NA-HPV and NA-PsV: If the relative infectivity value is $\leq 0.5$ compared with the negative control (representing a $50 \%$ or more reduction in infectivity), the serum is defined as seropositive.

†Kappa coefficients are used to assess the cross-reactivity, which is represented by the degree of agreement between two tests (HPV16 and HPV18). Kappa value ranges from 0 or $<0$ (a lack of agreement or a purely random coincidence) to 1 (a perfect agreement).

Table 1: Results of HPV16 or HPV18 antibody detected by the 3 assays among 94 HIV-infected women.

\begin{tabular}{|c|c|c|c|c|c|c|c|}
\hline VLP16-ELISA & NA-HPV16 & NA-PsV16 & $\mathrm{N}$ of women & VLP18-ELISA & NA-HPV18 & NA-PsV18 & $\mathrm{N}$ of women \\
\hline+ & + & + & 7 & + & + & + & 4 \\
\hline+ & + & - & 11 & + & + & - & 3 \\
\hline+ & - & + & 3 & + & - & + & 0 \\
\hline+ & - & - & 12 & + & - & - & 6 \\
\hline- & + & + & 9 & - & + & + & 11 \\
\hline- & + & - & 21 & - & + & - & 23 \\
\hline- & - & + & 6 & - & - & + & 5 \\
\hline- & - & - & 25 & - & - & - & 46 \\
\hline
\end{tabular}

+: Seropositive

-: Seronegative

Table 2: The distribution of seropositivity results across the 3 assays. 
Citation: Du P, Brendle S, Milici J, Camacho F, Zurlo J, et al. (2015) Comparisons of VLP-Based ELISA, Neutralization Assays with Native HPV, and Neutralization Assays with PsV in Detecting HPV Antibody Responses in HIV-Infected Women. J AIDS Clin Res 6: 433. doi:10.4172/21556113.1000433

Page 4 of 6

\begin{tabular}{|c|c|}
\hline Statistics & Measurement \\
\hline Pairwise Kappa coefficients and $\mathbf{9 5 \%} \mathbf{C l}^{*}$ & Agreement \\
\hline VLP16-ELISA vs. NA-HPV16 & $0.05(-0.14,0.24)$ \\
\hline VLP18-ELISA vs. NA-HPV18 & $0.06(-0.09,0.22)$ \\
\hline VLP16-ELISA vs. NA-PsV16 & $0.06(-0.14,0.26)$ \\
\hline VLP18-ELISA vs. NA-PsV18 & $0.09(-0.12,0.31)$ \\
\hline NA-HPV16 vs. NA-PsV16 & $0.14(-0.04,0.31)$ \\
\hline NA-HPV18 vs. NA-PsV18 & $0.29(0.11,0.46)$ \\
\hline Intraclass correlation coefficients $(I C C)^{\star *}$ & Consistency \\
\hline VLP16-ELISA, NA-HPV16, NA-PsV16 & ICC $=0.09$ \\
\hline VLP18-ELISA, NA-HPV18, NA-PsV18 & ICC $=0.18$ \\
\hline
\end{tabular}

*Kappa coefficients are used to assess the degree of agreement between the tests. Kappa value ranges from 0 or $<0$ (a lack of agreement or a purely random coincidence) to 1 (a perfect agreement)

**Intraclass correlation coefficients are used to measure the consistency of the results between the tests and to distinguish the results that could be highly correlated but with little agreement. The range of ICC is between 0 (no consistency) and 1 (perfect consistency).

Table 3: Agreement and consistency of the results between the 3 assays

and HPV18. There was an excellent reproducibility of the results in the NA-HPV (kappa=1.00 for HPV16 and kappa=0.77 for HPV18), but a low reproducibility in the VLP-ELISA (kappa=-0.17 for VLP16 and kappa $=0$ for VLP18) or the NA-PsV (kappa=-0.15 for PsV16 and kappa $=0$ for PsV18).

\section{Discussion}

We have found that the detection of HPV16/18 antibody responses varies across different assays in HIV-infected women. As the majority of women in our study acquired HIV via high-risk heterosexual contacts and more than $50 \%$ of women have a history of an abnormal cervical cytology, it is likely that some HIV-infected women were previously infected with HPV16 or 18 but cleared the infection. Because antibody responses following natural HPV infection is usually weak and not well correlated with the presence of HPV DNA [36], a sensitive and reliable approach is needed to define HPV seropositivity.

In our study the NA-HPV yields the highest seroprevalence for both HPV16 (51\%) and HPV18 (44\%) antibodies and also shows the lowest cross-reactivity (kappa $=0.04$ ) and an excellent reproducibility $(\mathrm{kappa}=1$ and 0.77$)$. Furthermore, the presence of HPV16 or HPV18 neutralizing antibody is observed in all HPV16- or HPV18-DNA positive women, suggesting that the NA-HPV is a sensitive method to study HPV serology. The results of the NA-HPV are unlikely caused by non-specific binding as the cross-reactivity between HPV16 and HPV18 is very low. If our study findings were due to non-specific reactivity or false positivity, we would observe a strong agreement between neutralizations with HPV16 and HPV18. The results from the NA-CRPV ( $4 \%$ seropositivity) also support the lack of non-specific neutralization.

The seroprevalence of HPV16 and 18 antibodies detected by the VLP-ELISA (35\% and 14\%, respectively) in our study are lower than the seroprevalence $(\sim 50 \%)$ reported in previous research [21,22]. Because the majority of our study population are women older than 40 years, the results could be due to the low levels of seroreactivity. We also performed the NA-PsV as PsVs are easy to produce and less expensive when compared to the native HPV. If the neutralization results are comparable, $\mathrm{PsV}$ could be used as a reasonable surrogate of native HPV for the neutralization assay. The NA-PsV yields an intermediate seropositivity compared with the VLP-ELISA and the NA-HPV, but the cross-reactivity and the reproducibility are similar to the VLP-ELISA.
We have also identified that the agreement between the three assays is generally low, although the direct comparison of the results may not be applicable because of different experimental designs and definitions of seropositivity. The inconsistent results between the three assays are likely caused by weak antibody responses following natural HPV infection in older women and different methods of the assays. In addition, HIV infection and the use of combined antiretroviral therapy might complicate HPV antibody responses and the natural history of HPV infection in HIV-infected women [37,38].

It has been increasingly recognized that HPV antibody responses vary across different assays [13,15-20]. The VLP-based assays are inexpensive, less labor-intensive, and multiple HPV VLP types can be tested simultaneously. However, only about $50 \%$ of HPV DNA positive people would be tested seropositive with the type-specific HPV antibody using a VLP-based assay [36]. Non-specific binding or binding on linear epitopes (not conformational epitopes) of the VLPs may also cause false seropositivity. Because VLPs are empty particles, the neutralization potential of HPV antibodies cannot be evaluated using the VLP-based assays. The NA-PsV has been shown as a better approach because the function of HPV antibodies can be assessed and the cross-reactivity or non-specific binding is generally low [12], thus, the NA-PsV is considered as the "gold standard" for measuring HPV neutralizing antibodies $[13,15,32]$. Nonetheless, PsVs differ in many characteristics from native HPV [27,39]. As both VLPs and PsVs are recombinant particles, denatured particles could cause false negative results and variable results, especially for unvaccinated people with low levels of seroreactivity $[7,8]$. The NA-HPV, mainly used in in vitro studies, can provide sensitive and reliable serology results. Another great advantage of the NA-HPV is that native HPVs are used to better understand host immunity against HPV infection. But due to unique technical requirements (the raft culture system) and labor intensity, the NA-HPV has not been utilized in population research. Given the merits of each assay, the selection of an appropriate assay is not obvious. The VLP-ELISA and NA-PsV are well established approaches to examine immune responses for vaccinated women with high titers of HPV antibodies, while the NA-HPV seems an appropriate method for HIVinfected women with low levels of HPV antibodies.

Several limitations need to be discussed when interpreting our study findings. First, in this exploratory study we have used different approaches for the VLP-based ELISA (CRPV VLP) and for the neutralization assay (NA-HPV) and our study population is older, thus, our results may not be comparable with previous findings. Second, as we focus on the overall seropositivity, we have not separated specific serum IgG, IgM and IgA antibody responses and not tested the titers of HPV antibodies with sequential dilutions of the serum. Last, because this study is a cross-sectional study with a small sample size, the results need to be confirmed with a larger scale study.

In conclusion, our study suggests that the NA-HPV appears to be a sensitive and reliable approach in detecting natural HPV antibodies in HIV-infected women, and also offers an opportunity to directly examine the function of HPV antibodies and measure the neutralization potential. Therefore, in addition to widely used VLPELISA and NA-PsV, the NA-HPV approach can be applied in HPV natural history studies or HPV vaccine studies to better understand host immunity against HPV infection in HIV population as well as in other populations.

\section{Acknowledgements}

The authors are very thankful to the Hershey Medical Center HIV Comprehensive Care Program treatment team members (Drs. Tonya Crook, 
Citation: Du P, Brendle S, Milici J, Camacho F, Zurlo J, et al. (2015) Comparisons of VLP-Based ELISA, Neutralization Assays with Native HPV, and Neutralization Assays with PsV in Detecting HPV Antibody Responses in HIV-Infected Women. J AIDS Clin Res 6: 433. doi:10.4172/21556113.1000433

Cynthia Whitener, Patsi Albright, and Daphne Greenawalt, Janice Powers, and Mary Catherine Hess) for patient recruitment and specimen collection.

\section{Sponsorship}

This study was funded by the NIH Career Development Program in Women's Health Research at Penn State (K12HD055882), Penn State College of Medicine, the Barsumian Trust, and the Pennsylvania Department of Health.

\section{Disclosure}

Dr. Du was supported by the National Institutes of Health $(\mathrm{NIH})$ grant nos. K12HD055882, "Career Development Program in Women's Health Research at Penn State" from the National Institute of Child Health and Human Development (NICHD), U54 HD34449. The content is solely the responsibility of the authors and does not necessarily represent the official views of the NICHD or the NIH.

Dr. Meyers has received speaker honoraria from Merck, Quest Diagnostics, GlaxoSmithKline, and Bristol-Myers. Dr. Meyers has performed research funded by Merck, The Phillip Morris External Research Program, NexMed, GlaxoSmithKline, OriGenix, and Interferon Sciences Inc.

Dr. Christensen has received royalties from Merck \& Co and GlaxoSmithKline.

\section{References}

1. Palefsky J (2007) Human papillomavirus infection in HIV-infected persons. Top HIV Med 15: 130-133.

2. Shiels MS, Pfeiffer RM, Gail MH, Hall HI, Li J, et al. (2011) Cancer burden in the HIV-infected population in the United States. J Natl Cancer Inst 103: 753-762.

3. Stanley M (2010) HPV-immune response to infection and vaccination. Infect Agent Cancer 5: 19.

4. Meyers C, Frattini MG, Hudson JB, Laimins LA (1992) Biosynthesis of human papillomavirus from a continuous cell line upon epithelial differentiation. Science 257: 971-973.

5. Kirnbauer R, Booy F, Cheng N, Lowy DR, Schiller JT (1992) Papillomavirus L1 major capsid protein self-assembles into virus-like particles that are highly immunogenic. Proc Natl Acad Sci U S A 89: 12180-12184.

6. Buck CB, Thompson CD, Pang YY, Lowy DR, Schiller JT (2005) Maturation of papillomavirus capsids. J Virol 79: 2839-2846.

7. Ferguson $M$, Wilkinson $D E$, Heath $A$, Matejtschuk $P(2011)$ The first internationa standard for antibodies to HPV 16. Vaccine 29: 6520-6526.

8. Bissett SL, Wilkinson D, Tettmar KI, Jones N, Stanford E, et al. (2012) Human papillomavirus antibody reference reagents for use in postvaccination surveillance serology. Clin Vaccine Immunol 19: 449-451.

9. Kirnbauer R, Hubbert NL, Wheeler CM, Becker TM, Lowy DR, et al. (1994) A virus-like particle enzyme-linked immunosorbent assay detects serum antibodies in a majority of women infected with human papillomavirus type 16 . J Natl Cancer Inst 86: 494-499.

10. Christensen ND, Dillner J, Eklund C, Carter JJ, Wipf GC, et al. (1996) Surface conformational and linear epitopes on HPV-16 and HPV-18 L1 virus-like particles as defined by monoclonal antibodies. Virology 223: 174-184

11. Dias D, Van Doren J, Schlottmann S, Kelly S, Puchalski D, et al. (2005) Optimization and validation of a multiplexed luminex assay to quantify antibodies to neutralizing epitopes on human papillomaviruses $6,11,16$, and 18. Clin Diagn Lab Immunol 12: 959-969.

12. Pastrana DV, Buck CB, Pang YY, Thompson CD, Castle PE, et al. (2004) Reactivity of human sera in a sensitive, high-throughput pseudovirus-based papillomavirus neutralization assay for HPV16 and HPV18. Virology 321: 205216

13. Dessy FJ, Giannini SL, Bougelet CA, Kemp TJ, David MP, et al. (2008) Correlation between direct ELISA, single epitope-based inhibition ELISA and pseudovirion-based neutralization assay for measuring anti-HPV-16 and antiHPV-18 antibody response after vaccination with the AS04-adjuvanted HPV16/18 cervical cancer vaccine. Hum Vaccin 4: 425-434

14. Wentzensen N, Rodriguez AC, Viscidi R, Herrero R, Hildesheim A, et al. (2011) A competitive serological assay shows naturally acquired immunity to human papillomavirus infections in the guanacaste natural history study. J Infect Dis 204: 94-102.

15. Krajden M, Cook D, Yu A, Chow R, Mei W, et al. (2011) Human papillomavirus 16 (HPV 16) and HPV 18 antibody responses measured by pseudovirus neutralization and competitive Luminex assays in a two- versus three-dose HPV vaccine trial. Clin Vaccine Immunol 18: 418-423.

16. Hernandez BY, Ton T, Shvetsov YB, Goodman MT, Zhu X (2012) Human papillomavirus (HPV) L1 and L1-L2 virus-like particle-based multiplex assays for measurement of HPV virion antibodies. Clin Vaccine Immunol 19: 13481352.

17. Safaeian M, Ghosh A, Porras C, Lin SW, Rodriguez AC, et al. (2012) Direct comparison of HPV16 serological assays used to define HPV-naive women in HPV vaccine trials. Cancer Epidemiol Biomarkers Prev 21: 1547-1554.

18. Faust H, Andersson K, Forslund O, Dillner J (2013) Pseudovirion-binding and neutralizing antibodies to cutaneous human papillomaviruses (HPV) correlated with the presence of HPV DNA in skin. J Gen Virol 94: 1096-1103.

19. Lin SW, Ghosh A, Porras C, Markt SC, Rodriguez AC, et al. (2013) HPV16 seropositivity and subsequent HPV16 infection risk in a naturally infected population: comparison of serological assays. PLoS One 8: e53067.

20. Scherpenisse M, Schepp RM, Mollers M, Mooij SH, Meijer CJ, et al. (2013) Comparison of different assays to assess human papillomavirus (HPV) type 16- and 18-specific antibodies after HPV infection and vaccination. Clin Vaccine Immunol 20: 1329-1332.

21. Petter A, Heim K, Guger M, Ciresa-Ko Nig A, Christensen N, et al. (2000) Specific serum IgG, IgM and IgA antibodies to human papillomavirus types $6,11,16,18$ and 31 virus-like particles in human immunodeficiency virusseropositive women. J Gen Virol 81: 701-708.

22. Viscidi RP, Snyder B, Cu-Uvin S, Hogan JW, Clayman B, et al. (2005) Human papillomavirus capsid antibody response to natural infection and risk of subsequent HPV infection in HIV-positive and HIV-negative women. Cancer Epidemiol Biomarkers Prev 14: 283-288.

23. Silverberg MJ, Schneider MF, Silver B, Anastos KM, Burk RD, et al. (2006) Serological detection of human papillomavirus type 16 infection in human immunodeficiency virus (HIV)-positive and high-risk HIV-negative women. Clin Vaccine Immunol 13: 511-519.

24. Heard I (2011) Human papillomavirus, cancer and vaccination. Curr Opin HIV AIDS 6: 297-302.

25. Wang X, Sapp M, Christensen ND, Dillner J (2005) Heparin-based ELISA reduces background reactivity in virus-like particle-based papillomavirus serology. J Gen Virol 86: 65-73.

26. Christensen ND, Hopfl R, DiAngelo SL, Cladel NM, Patrick SD, et al. (1994) Assembled baculovirus-expressed human papillomavirus type $11 \mathrm{~L} 1$ capsid protein virus-like particles are recognized by neutralizing monoclonal antibodies and induce high titres of neutralizing antibodies. J Gen Virol 75: 2271-2276.

27. Conway MJ, Cruz L, Alam S, Christensen ND, Meyers C (2011) Crossneutralization potential of native human papillomavirus $\mathrm{N}$-terminal L2 epitopes. PLoS One 6: e16405.

28. McLaughlin-Drubin ME, Christensen ND, Meyers C (2004) Propagation, infection, and neutralization of authentic HPV16 virus. Virology 322: 213-219.

29. Christensen ND, Kreider JW, Cladel NM, Galloway DA (1990) Immunological cross-reactivity to laboratory-produced HPV-11 virions of polysera raised against bacterially derived fusion proteins and synthetic peptides of HPV-6b and HPV-16 capsid proteins. Virology 175: 1-9.

30. Pfaffl MW (2001) A new mathematical model for relative quantification in realtime RT-PCR. Nucleic Acids Res 29: e45.

31. Schiller JT (2004) Lab of Cellular Oncology Technical Files.

32. World Health Organization (2010) Human papillomavirus laboratory manual First edition, 2009. Geneva, Switzerland.: World Health Organization.

33. Christensen ND, Kreider JW (1991) Neutralization of CRPV infectivity by monoclonal antibodies that identify conformational epitopes on intact virions. Virus Res 21: 169-179

34. Culp TD, Christensen ND (2003) Quantitative RT-PCR assay for HPV infection in cultured cells. J Virol Methods 111: 135-144

35. Culp TD, Christensen ND (2004) Kinetics of in vitro adsorption and entry of papillomavirus virions. Virology 319: 152-161.

36. Dillner J1 (1999) The serological response to papillomaviruses. Semin Cancer Biol 9: 423-430.

37. Marais DJ, Carrara H, Ramjee G, Kay P, Williamson AL (2009) HIV-1 
Citation: Du P, Brendle S, Milici J, Camacho F, Zurlo J, et al. (2015) Comparisons of VLP-Based ELISA, Neutralization Assays with Native HPV, and Neutralization Assays with PsV in Detecting HPV Antibody Responses in HIV-Infected Women. J AIDS Clin Res 6: 433. doi:10.4172/21556113.1000433

Page 6 of 6

seroconversion promotes rapid changes in cervical human papillomavirus (HPV) prevalence and HPV-16 antibodies in female sex workers. J Med Virol 81: $203-210$

38. Mitchell D, Israr M, Alam S, Kishel J, Dinello D, et al. (2012) Effect of the
HIV nucleoside reverse transcriptase inhibitor zidovudine on the growth and differentiation of primary gingival epithelium. HIV Med 13: 276-290.

39. Conway MJ, Meyers C (2009) Replication and assembly of human papillomaviruses. J Dent Res 88: 307-317. 